\title{
Exhaustivity in Topological Riesz Spaces with the Principal Projection Property \\ by
}

Kimberly MULLER

Presented by Stanistaw KWAPIEŃ

\begin{abstract}
Summary. Exhaustive and uniformly exhaustive elements are studied in the setting of locally solid topological Riesz spaces with the principal projection property. We study the structure of the order interval $[0, x]$ when $x$ is an exhaustive element and the structure of the solid hull of a set of uniformly exhaustive elements.
\end{abstract}

1. Introduction. In functional analysis there has been a large amount of study on the embeddability of the classical Banach spaces $c_{0}, \ell_{1}$ and $\ell_{\infty}$ in other Banach spaces. Because of results from vector measure theory such as the Diestel-Faires theorem [8], these studies are often done in conjunction with studies on strongly additive measures. In many of these studies the main emphasis is on normed vector spaces, or more specifically, Banach spaces. Although many of these spaces are partially ordered, less attention has been given to the properties that are inherent to the partial ordering on the space. In the 1940's mathematicians began studying these partially ordered vector spaces in more detail and many results have been obtained, especially in the study of Banach lattices. In this paper we specifically want to study the concepts of exhaustivity, (absolute) continuity, and strong additivity in the more general setting of topological Riesz spaces. Many of these results will generalize results known for Banach lattices.

As is pointed out in the introduction of [9], early interest in weak and weak* compactness was often motivated by vector measure theory. This is illustrated by the following two well-known results.

2000 Mathematics Subject Classification: 46A40, 46B20, 46B42.

Key words and phrases: uniform exhaustivity, continuity, principal projection property, Riesz space, locally solid topology. 
THEOREM 1.1. A set $K \subseteq \mathrm{ca}(\Sigma)$ is weakly sequentially compact iff it is bounded and the countable additivity of $\mu$ on $\Sigma$ is uniform for $\mu \in K$.

TheOrem 1.2. A set $K \subseteq \mathrm{ca}(\Sigma)$ is weakly sequentially compact iff it is bounded and, for some positive $\lambda \in \mathrm{ca}(\Sigma), \mu \ll \lambda$ uniformly for $\mu \in K$.

As the following theorems illustrate, there is a strong connection between strong additivity and countable additivity. These theorems can be extended to the setting of topological Riesz spaces. The extensions use the lattice properties of the space of vector-valued measures and minimize set-theoretic manipulations.

Theorem 1.3 (Bell-Bilyeu-Lewis [3]). A positive element $k$ of a $\sigma$ Dedekind complete Banach lattice $X$ is exhaustive if and only if the norm is countably order continuous on the order interval $[0, k]$.

TheOREM 1.4 (Brooks [5], Drewnowski [11]). Suppose $\left(\mu_{n}\right)$ is a sequence of countably additive scalar functions on the $\sigma$-algebra $\Sigma$, and $\mu$ is a finitely additive (possible infinite) measure on $\Sigma$ such that $\mu_{n} \ll \mu$ for each $n$. Then $\mu_{n} \ll \mu$ uniformly.

A recent extension of Theorem 1.4 has been made in the study of submeasures [13]. Also, Drewnowski and Labuda [12] proved a result similar to Theorem 1.3 for a disjointly $\sigma$-Dedekind complete TRS. Their methods were quite different due to the difference in hypotheses. Also the emphasis on exhaustivity in [12] is on vector measures and not exhaustive elements in a TRS. In [12], a characterization of exhaustive vector measures is made for Lebesgue and pre-Lebesgue topologies. That connection will also be made in the setting of this paper. For more on Lebesgue and pre-Lebesgue topologies see Aliprantis and Burkinshaw [1]. Also the question was raised in [3] of whether or not $[0, k]$ must be separable whenever $k$ is exhaustive. A counterexample will be provided in Section 3 .

2. Continuity in topological Riesz spaces. If $X$ is a Riesz space, $X$ is said to have the principal projection property (PPP) provided that for each pair of $x$ and $y$ in $X_{+}=\{z: z \geq 0\}$ the element $\bigvee_{n} n x \wedge y$ exists. This definition is equivalent to that found in [17]. If $X$ is a Riesz space with the PPP, define $P_{x}(y)=\bigvee_{n} n x \wedge y$ for all $x, y \in X_{+}$. For arbitrary $y \in X$ define $P_{x}(y)$ to be $P_{x}\left(y^{+}\right)-P_{x}\left(y^{-}\right)$. These projections have proved to be useful in many different areas. For instance, if $\mu$ and $\nu$ are scalar valued measures these projections can be used to find the absolutely continuous and singular parts of $\nu$ with respect to $\mu$ only using the order properties of the reals. These projections have also been applied to abstract L-spaces [14], measure spaces [4], and more recently, submeasures [13]. Note that if $x, y \in X_{+}$the 
following properties hold. The majority of them can be found in the results of Kakutani [14].

(a) $2 P_{x}(y) \wedge y=P_{x}(y)$.

(b) $P_{x}(y) \wedge\left(y-P_{x}(y)\right)=0$.

(c) $x \wedge\left(y-P_{x}(y)\right)=x \wedge y-x \wedge P_{x}(y)=0$.

(d) If $x=\psi+\eta$, where $\psi \wedge \eta=0$, then $P_{\psi}(y)+P_{\eta}(y)=P_{x}(y)=P_{\psi \vee \eta}(y)$.

(e) $P_{x}$ is linear.

(f) $P_{P_{x}(y)}=P_{x} P_{y}=P_{x \wedge y}$.

(g) $\left|P_{x}(y)\right|=P_{x}(|y|)$.

Let $O=\left\{P_{x}: x \in X_{+}\right\}$. A sequence $\left(P_{i}\right)$ from $O$ is said to be disjoint (or pairwise disjoint) if $P_{i} P_{j}=0$ for $i \neq j$. Assume that $(X, \tau)$ is a TRS with the PPP.

\section{DEFINITIONS.}

(1) A subset $K$ of $X$ is said to be (uniformly) continuous with respect to an element $m \in X$ if $P_{i}(u) \rightarrow 0$ (uniformly) for $u \in K$ whenever $P_{i}$ is a sequence from $O$ such that $P_{i}(m) \rightarrow 0$.

(2) A subset $K$ of $X$ is said to be (uniformly) exhaustive if $P_{i}(u) \rightarrow 0$ (uniformly) for $u \in K$ whenever $\left(P_{i}\right)$ is a disjoint sequence from $O$. If $K=\{k\}$ is a singleton we say that $k$ is exhaustive.

Note that if $X=\mathrm{ba}(\Sigma)$, then $\mu \in X$ is exhaustive if and only if it is strongly additive and $\mu$ is absolutely continuous with respect to $\nu \in X$ if and only if it is continuous with respect to $\nu$ using the above definition. The following lemmas will be helpful in establishing the main result of this section. The first lemma is true for any Riesz space with the PPP and can be found in [3]. For the remaining results we will assume that we have a TRS.

Lemma 2.1. Suppose $X$ is a Riesz space with the PPP. If $x$ and $y$ are in $X$ and $0 \leq y \leq x$, then there is a $z$ in $X_{+}$so that $P_{x}-P_{y}=P_{z}$.

Proof. Suppose the hypotheses are satisfied. From property (b) above we see that $P_{y}(x) \wedge\left(x-P_{y}(x)\right)=0$ and from $(\mathrm{d})$ we have $P_{x}=P_{P_{y}(x)}+P_{x-P_{y}(x)}$. Using property (f) and the fact that $y \leq x$ we obtain $P_{P_{y}(x)}=P_{x \wedge y}=P_{y}$. Therefore $P_{x}=P_{y}+P_{x-P_{y}(x)}$. Since $P_{y}(x) \leq x$ we have $P_{x}-P_{y} \in O$.

Lemma 2.2. Suppose $X$ is a TRS with the PPP. If $K$ is a uniformly exhaustive subset of $X$, then $|K|=\{|x|: x \in K\}$ is uniformly exhaustive.

Proof. Suppose $K$ is uniformly exhaustive. Suppose $\left(x_{n}\right)$ is a disjoint sequence from $X_{+}$. Let $V$ be a solid neighborhood of the origin. From (g) we have $\left|P_{x}(u)\right|=P_{x}(|u|)$ for all $u \in K$. Choose a natural number $N$ such that $P_{x_{n}}(u) \in V$ for every $n \geq N$ and every $u \in K$. Recall that $V$ is solid, $P_{x_{n}}(|u|)=\left|P_{x_{n}}(u)\right|$, and $P_{x_{n}}(u) \in V$. Therefore $P_{x_{n}}(|u|) \in V$ for all $n \in \mathbb{N}$ and all $u \in K$. Consequently, $|K|$ is uniformly exhaustive. 
TheOREm 2.3. Suppose that $X$ is a TRS with the PPP and $K$ is a subset of $X$. If $K$ is a uniformly exhaustive subset of $X$ and $\left(P_{x_{i}}\right)$ is a sequence from $O$, then for every solid neighborhood $V$ of the origin there exists a natural number $N$ so that $\left(P_{x_{k}}-P_{x_{k} \wedge \bigvee_{i=1}^{n} x_{i}}\right)(u) \in V$ for all $u \in K$ whenever $k \geq n \geq N$.

Proof. Suppose the conclusion is false. Then there is a solid neighborhood $V$ of the origin, an increasing sequence $\left(n_{i}\right)$ of positive integers, and a sequence $\left(u_{i}\right)$ from $K$ so that for all $i,\left(P_{x_{n_{i}}}-P_{x_{n_{i}} \wedge \bigvee_{k=1}^{n_{i-1}} x_{k}}\right)\left(u_{i}\right)$ is not in $V$. A calculation using properties (c), (d), and (f) shows that the above projections are pairwise disjoint. Lemma 2.1 shows the projections are in $O$. This contradicts the uniform exhaustivity of $K$.

The following theorem is the main result of this section. If $X$ is a Banach lattice and $|x| \leq|y|$, we have by definition $\|x\| \leq\|y\|$. In many cases when working with Banach lattices, it is this property of the norm that is used and not the fact that the norm is complete. In particular, if $X$ is a TRS and $y$ belongs to a locally solid neighborhood $V$ of the origin, then $x \in V$ whenever $|x| \leq|y|$. This property facilitates the proof of the following generalization of Theorem 1.4. Theorem 1.4 was established independently by L. Drewnowski [11] and James K. Brooks [5]. This argument also simplifies arguments in [6].

TheOREM 2.4. Suppose that $X$ is a metrizable TRS with the PPP and $K$ is a uniformly exhaustive subset of $X$. If $K$ is continuous with respect to $m \in X_{+}$, then $K$ is uniformly continuous with respect to $m$.

Proof. Suppose that $\varrho$ is a metric for the TRS $X$. Further suppose that $m \in X_{+}$and that $K$ is a uniformly exhaustive subset of $X$ so that $K$ is continuous with respect to $m$ but not uniformly continuous with respect to $m$. Then there exists a sequence $x_{i}$ from $X_{+}$, a locally solid neighborhood $V$ of the origin, and a sequence $y_{i}$ from $K$ so that $P_{x_{i}}(m) \rightarrow 0$ and

$$
P_{x_{i}}\left(y_{i}\right) \notin 2 V \quad \text { for all } i \text {. }
$$

We can assume without loss of generality that

$$
\sum \varrho\left(0, P_{x_{i}}(m)\right)<\infty
$$

Applying Theorem 2.3, let $n_{1}$ be a positive integer so that if $n \geq n_{1}$ then

$$
\left(P_{x_{n}}-P_{x_{n} \wedge \bigvee_{k=1}^{n_{1}} x_{k}}(u)\right) \in \frac{1}{2} V \quad \text { for all } u \in K .
$$

Let $z_{1}=\bigvee_{k=1}^{n_{1}} x_{k}$. Then from (I) and the statement above we find that $P_{z_{1} \wedge x_{n}}\left(y_{n}\right)$ is not in $\left(2-\frac{1}{2}\right) V$ for all $n \geq n_{1}$. Now let $a_{1}=z_{1} \wedge x_{n_{1}}, a_{2}=$ $z_{1} \wedge x_{n_{1}+1}, \ldots$ Applying Theorem 2.3 to $\left(P_{a_{i}}\right)$, let $n_{2}\left(>n_{1}\right)$ be a positive 
integer so that if $n \geq n_{2}$, then

$$
\left(P_{a_{n}}-P_{a_{n} \wedge \bigvee_{k=1}^{n_{2}} a_{k}}(u)\right) \in \frac{1}{4} V \quad \text { for all } u \in K .
$$

Let $z_{2}=\bigvee_{k=1}^{n_{2}} a_{k}$. By a similar argument to that above, there is a sequence $\left(b_{n}\right)$ in $K$ such that $P_{z_{2} \wedge a_{n}}\left(b_{n}\right)$ is not in $\left(2-\frac{1}{2}-\frac{1}{4}\right) V$ for all $n \geq n_{2}$. Since $\left(2-\frac{1}{2}-\frac{1}{4}\right) V$ is locally solid and $\left|P_{z_{2}}\left(b_{n}\right)\right|=P_{z_{2}}\left(\left|b_{n}\right|\right) \geq P_{a_{n} \wedge z_{2}}\left(b_{n}\right)$, we see that $\left|P_{z_{2}}\left(b_{n}\right)\right|$ is not in $\left(2-\frac{1}{2}-\frac{1}{4}\right) V$ for all $n \geq n_{2}$.

Continue inductively to manufacture a sequence $\left(z_{k}\right)$ from $X_{+}$and a subsequence $\left(d_{k}\right)$ of $\left(y_{n}\right)$ such that

$$
\begin{aligned}
& z_{k+1} \leq z_{k}, \\
& P_{z_{k}}\left(\left|d_{k}\right|\right) \notin V \quad \text { for each } k .
\end{aligned}
$$

Next observe that if $\left\{q_{1}, \ldots, q_{t}\right\} \subseteq X_{+}, u \in X_{+}$, and $w=\bigvee q_{i}$, then $P_{w}(u) \leq$ $\sum_{i=1}^{t} P_{q_{i}}(u)$. Therefore, using (II) and the fact that the sequence $\left(z_{k}\right)$ was defined inductively in terms of $\left(x_{k}\right)\left(z_{k} \leq \bigvee_{i=n_{k-1}}^{n_{k}} x_{i}\right)$, we get

$$
P_{z_{k}}(m) \rightarrow 0 \text {. }
$$

Now use (IV), (V), and the fact that $K$ (and therefore $|K|$ ) is continuous with respect to $m$ to select subsequences $\left(P_{z_{k_{i}}}\right)$ of $\left(P_{z_{k}}\right)$ and $\left(d_{k_{i}}\right)$ of $\left(d_{k}\right)$ such that

$$
\left(P_{z_{k_{i}}}-P_{z_{k_{i+1}}}\right)\left(\left|d_{k_{i}}\right|\right) \notin \frac{1}{2} V \quad \text { for each } i \text {. }
$$

But (III) implies this sequence of differences of projections is a disjoint sequence from $O$. This contradicts the uniform exhaustivity of $|K|$.

3. Exhaustivity. The results of this section further characterize exhaustivity in a TRS. If $A$ is a subset of $X$, then $\widehat{A}$ denotes the solid hull of $A$, i.e. $\widehat{A}=\{y \in X:|y| \leq|x|$ for some $x \in A\}$. The next theorem describes the structure of $\widehat{K}$ when $K$ is a uniformly exhaustive subset of $X$. Recall that an ideal in a TRS $X$ is a solid vector subspace of $X$.

Theorem 3.1. Suppose $X$ is a TRS with the PPP. A subset $K$ of $X$ is uniformly exhaustive iff each disjoint sequence in $\widehat{K}$ converges to zero. Furthermore, if $I$ is an ideal in $X$ and $K$ is a subset of $I$ so that $P_{x_{i}}(k) \rightarrow 0$ uniformly for $k \in K$ whenever $\left(x_{i}\right)$ is a disjoint sequence from $I_{+}$, then $K$ is uniformly exhaustive in $X$.

Proof. First suppose $K$ is uniformly exhaustive and $\left(x_{i}\right)$ is a disjoint sequence from $\widehat{K}\left(\left|x_{i}\right| \wedge\left|x_{j}\right|=0\right.$ if $\left.i \neq j\right)$. For each $i$ choose $y_{i} \in K$ so that $\left|x_{i}\right| \leq\left|y_{i}\right|$. Since $P_{u}(u)=u$ for all $u \in X$ and $P_{x_{i}}$ is monotone on $X_{+}$, it follows that

$$
0 \leq\left|x_{i}\right|=P_{\left|x_{i}\right|}\left(\left|x_{i}\right|\right) \leq P_{\left|x_{i}\right|}\left(\left|y_{i}\right|\right)=\left|P_{\left|x_{i}\right|}\left(y_{i}\right)\right|
$$


Since $\left(x_{i}\right)$ is a disjoint sequence, property (f) ensures $\left(P_{\left|x_{i}\right|}\right)$ is a disjoint sequence from $O$. Suppose $V$ is a solid neighborhood of the origin. Choose a natural number $N$ so that for every $i \geq N, P_{\left|x_{i}\right|}(y) \in V$ for all $y \in K$. Then clearly $P_{\left|x_{i}\right|}\left(y_{i}\right) \in V$ for all $i \geq N$. Since $V$ is solid we infer that $x_{i}$ is in $V$ for all $i \geq N$. Consequently, $x_{n} \rightarrow 0$.

Conversely, suppose that each disjoint sequence in $\widehat{K}$ converges to zero. Suppose $P_{x_{i}}$ is a disjoint sequence from $O$, and $\left(\left|u_{i}\right|\right)$ is a sequence from $\widehat{K}$. Then $\left(x_{i}\right)$ is a disjoint sequence from $X_{+}$and $P_{x_{i}}\left(\left|u_{i}\right|\right)$ is a disjoint sequence. Since $P_{x_{i}}\left(\left|u_{i}\right|\right) \leq\left|u_{i}\right|$ and $\left|u_{i}\right|$ is in $\widehat{K}$, we have $P_{x_{i}}\left(\left|u_{i}\right|\right) \in \widehat{K}$. Thus $P_{x_{i}}\left(\left|u_{i}\right|\right) \rightarrow 0$. Again using property $(\mathrm{g})$ and the fact that $X$ has a locally solid topology, we find that $P_{x_{i}}\left(u_{i}\right) \rightarrow 0$. Since the choice of the sequence $\left|u_{i}\right|$ from $\widehat{K}$ was arbitrary, it follows that $K$ is uniformly exhaustive.

Next suppose $I$ is an ideal in $X, K$ is a subset of $I$ satisfying the hypotheses of the final statement of the theorem, and $\left(\psi_{i}\right)$ is a disjoint sequence in $X_{+}$. Let $\left(u_{i}\right)$ be an arbitrary sequence in $K$. Then $\psi_{i} \wedge\left|u_{i}\right| \in I$ for all $i$ and $\left(\psi_{i} \wedge\left|u_{i}\right|\right)$ is a disjoint sequence. By the hypothesis $P_{\psi_{i} \wedge\left|u_{i}\right|}\left(\left|u_{i}\right|\right) \rightarrow 0$. Also

$$
P_{\psi_{i}}\left(\left|u_{i}\right|\right)=\bigvee n \psi_{i} \wedge\left|u_{i}\right|=\bigvee\left[n\left(\psi_{i} \wedge\left|u_{i}\right|\right) \wedge\left|u_{i}\right|\right]=P_{\psi_{i} \wedge\left|u_{i}\right|}\left(\left|u_{i}\right|\right)
$$

Since $X$ has a locally solid topology, $P_{\psi_{i}}\left(\left|u_{i}\right|\right) \rightarrow 0$.

The next main result characterizes exhaustivity in a TRS with the PPP. The final statement of Theorem 3.3 is similar to the results in [12] for vector measures. However, the initial statement of Theorem 3.3 sheds light on the structure of the order interval $[0, k]$ when $k$ is exhaustive. This theorem is closely akin to some of the major theorems on Banach lattices in Section 5 of Chapter 2 of [19]. If a space $X$ is not pre-Lebesgue, the exhaustive elements in $X$ are often of interest. Note that the set of exhaustive elements of $X=l_{\infty}$ is $c_{0}$. In order to prove Theorem 3.3, we use the following lemma, which can be found in [16]. It is a generalization of the Meyer-Nieberg lemma found on page 92 of [19].

Lemma 3.2. Let $(X, \tau)$ be a locally solid topological Riesz space. Suppose that $\nu_{n}$ is a sequence in $X_{+}$with $\nu_{n} \nrightarrow 0$ and $\left\{\sum_{i=1}^{n} \nu_{i} \mid n \in \mathbb{N}\right\}$ bounded. Suppose further that one of the following conditions is satisfied:

(i) $\left(\nu_{n}\right)$ is majorized by some $x \in X_{+}$.

(ii) $X$ is Dedekind $\sigma$-complete with an order continuous topology on $\left[0, \nu_{n}\right]$ for each $n$.

Then there exists a sequence $(k(n))$ of natural numbers and a disjoint sequence $\left(x_{n}\right)$ in $X_{+}$so that $x_{n} \nrightarrow 0$ and $x_{n} \leq \nu_{k(n)}$ for every $n$. 
Theorem 3.3. Suppose $X$ is a TRS with the PPP. A positive element $k$ is exhaustive iff $\left(u_{i}\right)$ is Cauchy whenever $0 \leq u_{i} \uparrow \leq k$. Consequently, $X$ is pre-Lebesgue iff every positive element of $X$ is exhaustive.

Proof. Suppose $0 \leq u_{i} \uparrow \leq k$ implies $\left(u_{n}\right)$ is Cauchy, and suppose $k$ is not exhaustive. Then there is a disjoint sequence $\left(x_{n}\right)$ from $X_{+}$and a solid neighborhood $V$ of the origin so that $P_{x_{i}}(k) \notin V$ for all $i \in \mathbb{N}$. Let

$$
u_{n}=\sum_{i=1}^{n} P_{x_{i}}(k)=P_{\sum_{i=1}^{n} x_{i}}(k) \leq k .
$$

Then $0 \leq u_{i} \uparrow \leq k$. By our hypothesis, $\left(u_{n}\right)$ is a Cauchy sequence. Choose an $N$ so that if $m, n \geq N$, then $u_{n}-u_{m} \in V$. If $n \geq m$, then $u_{n}-u_{m}=$ $\sum_{i=m+1}^{n} P_{x_{i}}(k) \in V$. Since $V$ is solid, $P_{x_{n}}(k) \in V$, which contradicts the assumption.

Conversely, suppose $k$ is exhaustive and suppose there is a sequence $\left(u_{n}\right)$ so that $0 \leq u_{i} \uparrow \leq k$ and $\left(u_{n}\right)$ is not Cauchy. Choose a solid neighborhood $V$ of the origin and intertwining sequences $\left(n_{i}\right)$ and $\left(m_{i}\right)$ so that $y_{i}=u_{n_{i}}-u_{m_{i}}$ $\notin V$ for each $i$. Then for all $n, \sum_{i=1}^{n} y_{i} \leq k$. Now apply Lemma 3.2 to find a disjoint sequence $\left(x_{n}\right)$ in $X_{+}$and $(k(n))$ so that $x_{n} \notin V$ for all $n$ and $x_{n} \leq y_{k(n)} \leq k$. Consequently, $P_{x_{n}}(k) \geq P_{x_{n}}\left(x_{n}\right)=x_{n} \notin V$ and $k$ is not exhaustive.

We can now use the above theorem to establish a generalization of Theorem 1.3. It is known that $X$ is Lebesgue iff each element of $X$ is exhaustive whenever $X$ is a sequentially complete and Dedekind $\sigma$-complete TRS. The following result investigates the structure of the order interval $[0, k]$ if $k$ is exhaustive.

Corollary 3.4. Suppose $X$ is a sequentially complete and Dedekind $\sigma$ complete TRS. A positive element $k$ is exhaustive iff $y_{i} \rightarrow 0$ whenever $y_{i} \downarrow 0$ in the order interval $[0, k]$.

Proof. Suppose $k$ is exhaustive and $\left(y_{n}\right)$ is a sequence from $[0, k]$ so that $y_{n} \downarrow 0$. Then $k-y_{n}$ is an increasing sequence in $[0, k]$. By Theorem $3.3, k-y_{n}$ is Cauchy. Since $X$ is sequentially complete, $k-y_{n}$ converges. Therefore $\left(y_{n}\right)$ converges. Since $y_{n} \downarrow 0$, we have $y_{n} \rightarrow 0$.

Now suppose for every sequence $\left(y_{n}\right)$ in $[0, k]$ with $y_{n} \downarrow 0$ we have $y_{n} \rightarrow 0$. Suppose that $k$ is not exhaustive. Then there is a disjoint sequence $\left(P_{x_{i}}\right)$ from $O$ and a solid neighborhood $V$ of the origin so that $P_{x_{i}}(k) \notin V$ for each $i$. Let $l_{n}=P_{\bigvee_{i=1}^{n} x_{i}}(k)$ and $l=\bigvee_{n} l_{n}$. Then $l-l_{n} \downarrow 0$. By our assumption $l-l_{n} \rightarrow 0$. Then $l_{n}$ is a Cauchy sequence. However, $l_{n+1}-l_{n}=P_{x_{n+1}}(k) \notin V$. Therefore we have a contradiction and $k$ is exhaustive.

When studying uniform absolute continuity and uniform exhaustivity, it is natural to consider the following two classical results from measure theory. 
Theorem 3.5 (Vitali-Hahn-Saks). Let $(S, \Sigma, \mu)$ be a measure space and $\left(\lambda_{n}\right)$ a sequence of $\mu$-continuous vector or scalar valued additive set functions on $\Sigma$. If the limit $\lim \lambda_{n}(E)$ exists for each $E$ in $\Sigma$ then $\lambda_{n}$ is uniformly absolutely continuous with respect to $\mu$.

ThEOREM 3.6 (Brooks-Jewett, [6]). Let $\left(\lambda_{n}\right)$ be a sequence of strongly additive vector or scalar valued set functions defined on $\Sigma$. If the limit $\lim \lambda_{n}(E)$ exists for each $E$ in $\Sigma$, then the sequence $\left(\lambda_{n}\right)$ is uniformly exhaustive.

Recall that in $\mathrm{ca}(\Sigma)$ weak convergence and setwise convergence are equivalent. Consider the unit vector basis $\left(e_{i}\right)$ in $c_{0}$. Each $e_{i}$ is exhaustive and $\left(e_{i}\right)$ is weakly convergent, but $P_{e_{i}}\left(e_{i}\right)=e_{i}$, which implies $\left(e_{i}\right)$ is not uniformly exhaustive. Therefore, the Brooks-Jewett theorem does not hold in arbitrary Banach lattices if we replace setwise convergence with weak convergence. If $X$ is a Schur space then weak and norm convergence will coincide. Therefore in a Schur space, the weak convergence of the sequence $\left(x_{i}\right)$ is sufficient to guarantee that the sequence is uniformly exhaustive. Again consider $\left(e_{i}\right)$ in $c_{0}$ and let $x=(1 / n)_{n}$ be in $c_{0}$. Each $e_{i}$ is continuous with respect to $x$. However, $P_{e_{i}}(x) \rightarrow 0$ and $P_{e_{i}}\left(e_{i}\right)=e_{i} \nrightarrow 0$. Therefore the continuity is not uniform. Thus the Vitali-Hahn-Saks theorem does not hold in arbitrary Banach lattices if we replace setwise convergence with weak convergence.

Now note that by combining Lemma II.5.4 of [19], and Theorem 3.1 and Corollary 3.4 above, we find that a positive element $x$ of the Dedekind $\sigma$-complete Banach lattice $X$ is exhaustive whenever $[0, x]$ is separable. This is due to the fact that separability of the order interval $[0, x]$ yields the separability of the principal ideal $I_{x}$ generated by $x$. Therefore $I_{x}$ is Lebesgue and each element of $I_{x}$ is exhaustive. The question was raised in [3] of whether or not it is also true that $[0, x]$ is separable whenever $x$ is exhaustive. The following counterexample resolves this question.

Let $\mathbb{R}$ represent the real numbers. Also let $\lambda$ represent Lebesgue measure on the interval $[0,1]$. Using the results of Kakutani in [15], we can define a countably additive measure $m$ on the product space $([0,1], \lambda)^{\mathbb{R}}$. Let $\Sigma$ denote the measurable sets in $([0,1], \lambda)^{\mathbb{R}}$. Then $m$ is an exhaustive element of ca $(\Sigma)$. However, $[0, m]$ is not separable in $\mathrm{ca}(\Sigma)$. To see this let $m_{\alpha}=\left.m\right|_{E_{\alpha}}$ where $E_{\alpha}$ is the element of $\Sigma$ whose $\alpha$ th projection is $[0,1 / 2]$ and whose $\beta$ th projection is $[0,1]$ if $\alpha \neq \beta$. Therefore we have an uncountable number of elements of $[0, m]$ with $\left\|m_{\gamma}-m_{\beta}\right\| \geq 1 / 4$ whenever $\gamma \neq \beta$. Consequently, $[0, m]$ is not separable.

Finally, Theorem 2.4(vi) in [3] is false as stated. It states that if $X$ is a Dedekind $\sigma$-complete Banach lattice and $K$ is a subset of the exhaustive elements of $X$, then $K$ is continuous with respect to some exhaustive element 
in $X$ iff each pairwise disjoint subset of $K$ is countable. For a counterexample again let $\mathbb{R}$ be the set of real numbers and let $X=l_{\infty}(\mathbb{R})$. Let $K=\left\{e_{0}+e_{\alpha}\right.$ : $\alpha \in \mathbb{R}\}$. Then each element of $K$ is exhaustive. Vacuously, each disjoint subset of $K$ is countable. However, $K$ is not continuous with respect to any exhaustive element of $l_{\infty}(\mathbb{R})$. The theorem should have read as follows: If $X$ is a Dedekind $\sigma$-complete Banach lattice and $K$ is a subset of the exhaustive elements of $X$, then $K$ is continuous with respect to some exhaustive element in $X$ iff each pairwise disjoint subset of $\widehat{K}$ is countable. The proof runs as before.

\section{References}

[1] C. Aliprantis and O. Burkinshaw, Locally Solid Riesz Spaces, Academic Press, New York, 1978.

[2] -, -, Positive Operators, Academic Press, New York, 1985.

[3] W. Bell, R. Bilyeu and P. Lewis, Uniform exhaustivity and Banach lattices, Ann. Mat. Pura Appl. 4 (1944), 57-74.

[4] R. Bilyeu and P. Lewis, Uniform differentiability, uniform absolute continuity, and the Vitali-Hahn Saks theorem, Rocky Mountain J. Math. 10 (1980), 533-557.

[5] J. Brooks, Equicontinuous sets of measures and applications to Vitali's integral convergence theorem and control measures, Ad. Math. 10 (1973), 165-171.

[6] J. Brooks and R. Jewett, On finitely additive vector measures, Proc. Nat. Acad. Sci. U.S.A. 67 (1970), 1294-1298.

[7] J. Brooks and P. Lewis, Linear operators and vector measures, Trans. Amer. Math. Soc. 192 (1974), 139-162.

[8] J. Diestel and B. Faires, On vector measures, ibid. 198 (1974), 253-271.

[9] J. Diestel and J. Uhl, Vector Measures, Math. Surveys 15, Amer. Math. Soc., Providence, 1977.

[10] L. Drewnowski, Equivalence of Brooks-Jewett, Vitali-Hahn-Saks and Nikodym theorems, Bull. Acad. Polon. Sci. Sér. Sci. Math. Astronom. Phys. 20 (1972), $725-731$.

[11] -, Topological rings of sets, continuous set functions, integration. II, ibid. 80 (1972), $277-286$.

[12] L. Drewnowski and I. Labuda, Copies of $c_{0}$ and $\ell_{\infty}$ in topological Riesz spaces, Trans. Amer. Math. Soc. 350 (1998), 3555-3570.

[13] C. Huff and P. Lewis, Exhaustive submeasures on boolean rings, Banach lattices, and uniform absolute continuity, Bull. Polish Acad. Sci. Math. 49 (2001), 203-210.

[14] S. Kakutani, Concrete representation of abstract (L)-spaces, Ann. of Math. 42 (1941), 523-537.

[15] - Notes on infinite product measure spaces II, Proc. Imp. Acad. Tokyo 19 (1943), $184-188$.

[16] P. Lewis and K. Muller, Isomorphic embeddings and strongly additive measures, Monatsh. Math. 143 (2004), 21-33.

[17] W. Luxemburg and A. Zaanen, Riesz Spaces, Vol. 1, North-Holland, Amsterdam, 1971.

[18] P. Meyer-Nieberg, Zur schwachen Kompaktheit in Banachverbänden, Math. Z. 134 (1973), 303-315. 
[19] H. Schaefer, Banach Lattices and Positive Operators, Springer, New York, 1974.

Kimberly Muller

Department of Mathematics

Lake Superior State University

Sault Sainte Marie, MI 49783, U.S.A.

E-mail: kmuller@lssu.edu

Received March 31, 2005 